Cahiers
de a Recherche
sur les Droits Fondamentaux

\section{Cahiers de la recherche sur les droits} fondamentaux

$14 \mid 2016$

Urbanisme et droits fondamentaux

\title{
La Convention du Conseil de l'Europe contre le trafic d'organes humains : un instrument révolutionnaire pour la prévention et la répression du commerce illicite de la personne humaine
}

The Convention of the Council of Europe to Counter the Trafficking in Human Organs: A Revolutionary Tool to Prevent and to Fight against the Illicit

Trafficking in Humans

\section{Mamoud Zani}

\section{(2) OpenEdition}

\section{Journals}

Édition électronique

URL : https://journals.openedition.org/crdf/596

DOI : $10.4000 /$ crdf.596

ISSN : 2264-1246

Éditeur

Presses universitaires de Caen

\section{Édition imprimée}

Date de publication : 1 novembre 2016

Pagination : 101-107

ISBN : 978-2-84133-838-2

ISSN : $1634-8842$

Référence électronique

Mamoud Zani, «La Convention du Conseil de l'Europe contre le trafic d'organes humains : un instrument révolutionnaire pour la prévention et la répression du commerce illicite de la personne humaine », Cahiers de la recherche sur les droits fondamentaux [En ligne], 14 | 2016, mis en ligne le 01 octobre 2019, consulté le 16 novembre 2022. URL : http://journals.openedition.org/crdf/596 ; DOI https://doi.org/10.4000/crdf.596 


\title{
La Convention du Conseil de l'Europe contre le trafic d'organes humains: un instrument révolutionnaire pour la prévention et la répression du commerce illicite de la personne humaine
}

\author{
Mamoud ZANI \\ Professeur de droit public à la faculté de droit de Tunis \\ Directeur du Centre de droit international et européen (CDIE) de Tunis
}

I. Les aspects originaux de la Convention

A. La pénalisation des actes illicites de trafic d'organes humains

B. Les mesures de protection et de prévention des victimes

II. Le suivi fragile de l'application de la Convention
A. La pluralité des mécanismes de suivi
B. Les déficiences afférentes au système de suivi

Le trafic d'organes humains (TOH) constitue un crime transnational portant atteinte à la personne humaine et sa dignité. Ce fléau multinational ne date point d'aujourd'hui; en effet, il a été intrinsèquement lié au phénomène du tourisme de transplantation d'organes dénoncé en 2004 par l'Organisation mondiale de la santé ${ }^{1}(\mathrm{OMS})$, ainsi que par les États participants au sommet d'Istanbul (Turquie) de $2008^{2}$ concernant le trafic d'organes et le tourisme de transplantation.
Pour prendre à bras-le-corps ce problème de dimension mondiale, les organes statutaires du Conseil de l'Europe, principalement le Comité des ministres (organe décisionnel) et l'Assemblée parlementaire (organe représentatif), ont adopté un corpus normatif saisissant en vue de consolider l'action de l'institution strasbourgeoise pour une lutte efficace contre le trafic d'organes humains sous tous ses aspects. Au demeurant, il suffit pour s'en convaincre de mentionner, à titre illustratif, les normes

1. Voir la résolution WHA57.18 de l'Assemblée mondiale de la santé intitulée "Transplantation d'organes et de tissus humains", adoptée le 22 mai 2004, à l'occasion de sa $57^{\mathrm{e}}$ session (point 12.14 de l'ordre du jour). En vertu de l'article 1, alinéa 5, de la résolution, les États membres sont invités à "prendre des mesures pour protéger les plus pauvres et les groupes vulnérables du "tourisme de la transplantation" et de la vente de tissus et d'organes, en s'intéressant notamment au problème plus vaste du trafic international de tissus et d'organes humains ».

2. Tenu du 30 avril au 2 mai 2008, le sommet a abouti à l'adoption de la Déclaration d'Istanbul contre le trafic d'organes et le tourisme de transplantation composée d'un certain nombre de principes. Suivant le principe 6 de la Déclaration, «Le trafic d'organes et le tourisme de transplantation violent les principes d'égalité, de justice, et de respect de la dignité humaine et devraient être interdits. Le commerce de transplantation devrait être interdit parce qu'il mène inexorablement à l'inégalité et à l'injustice, dans la mesure où il a pour cible des donneurs vulnérables, qu'ils soient pauvres ou non". 
déclaratoires (recommandations) et les normes obligatoires (conventions) ci-après :

- Recommandation Rec (2001) 5 du Comité des ministres aux États membres sur la gestion des listes d'attente et des délais d'attente en matière de transplantation d'organe ${ }^{3}$ où il est précisé que «les États membres devraient garantir l'existence d'un système permettant d'assurer aux patients un accès équitable aux services de transplantation, de manière à ce que les organes et les tissus soient attribués conformément à des règles transparentes et dûment justifiables au regard de critères médicaux » (principe 1);

- Recommandation Rec (2003) 12 du Comité des ministres aux États membres relative aux registres des donneurs d'organes ${ }^{4}$; ces derniers sont tenus «d'envisager sérieusement la nécessité, et la finalité, d'un registre des donneurs d'organes" (principe 1);

- Recommandation 1611 (2003) 1 de l'Assemblée parlementaire sur le trafic d'organes en Europe ${ }^{5}$ invitant les États membres «à reconnaître leur responsabilité commune en matière de lutte contre le trafic d'organes, en renforçant les mécanismes de coopération déjà en place au niveau du Conseil de l'Europe - par le Comité d'experts sur les aspects organisationnels de la coopération en matière de transplantation d'organes (SP-CTO) - et en augmentant le budget consacré aux activités d'assistance menées dans ce domaine, qui sont un moyen essentiel de favoriser la création de systèmes de transplantation efficaces » $(\$ 14 \mathrm{C})$;

- Recommandation Rec (2004) 7 du Comité des ministres aux États membres sur le trafic d'organes ${ }^{6}$ par laquelle ceux-ci «doivent protéger toutes les personnes dans leur dignité et leur identité et leur garantir, sans discrimination, leurs droits et libertés fondamentales dans le domaine de la transplantation d'organes et de tissus» (art. 1);

- Recommandation 2009 (2013) intitulée «Vers une convention du Conseil de l'Europe pour lutter contre le trafic d'organes, de tissus et de cellules d'origine humaine $»^{7}$, par laquelle l'Assemblée parlementaire «note que les questions relatives à la prévention du trafic d'organes, à la protection des victimes et à la coopération nationale et internationale pour combattre ce trafic ne sont pas suffisamment développées [...]» (principe 4);

- Convention sur les droits de l'homme et la biomédecine du 4 avril 1997 (Convention d'Oviedo) ${ }^{8}$ dont la finalité consiste à "protége [r] l'être humain dans sa dignité et son identité et garanti[r] à toute personne, sans discrimination, le respect de son intégrité et de ses autres droits et libertés fondamentales à l'égard des applications de la biologie et de la médecine» (chap. I: «Dispositions générales», art. 1);

- Convention du Conseil de l'Europe sur la lutte contre la traite des êtres humains du 16 mai $2005^{9}$ qui vise à «protéger les droits de la personne humaine des victimes de la traite, [à] concevoir un cadre complet de protection et d'assistance aux victimes et aux témoins, en garantissant l'égalité entre les femmes et les hommes, ainsi qu['à] assurer des enquêtes et des poursuites efficaces» (art. 1, al. b).

En dépit de l'importance de cet arsenal normatif diversifié, il semble que la totalité des normes produites a démontré ses limites pour venir concrètement à bout de la question du trafic d'organes humains. D'où l'importance pour le Conseil de l'Europe d'élaborer un instrument juridique contraignant spécifique audit trafic, surtout que le champ d'application des deux conventions citées ci-devant ne peut couvrir directement la problématique du trafic d'organes humains.

À la suite de l'étude conjointe menée en 2009 par l'Organisation des Nations unies et le Conseil de l'Europe sur le trafic d'organes, de tissus et de cellules et la traite des êtres humains aux fins de prélèvement d'organes ${ }^{10}$, préconisant, entre autres, la préparation d'un

[...] instrument juridique international établissant une définition du trafic d'OTC et énonçant des mesures à prendre pour prévenir ce trafic et protéger ses victimes, ainsi que des mesures de droit pénal destinées à le réprimer ${ }^{11}$

le Comité des ministres du Conseil de l'Europe chargea ${ }^{12}$ respectivement, le 16 novembre 2010, le Comité européen pour les problèmes criminels (CDPC), le Comité directeur

3. Adoptée le 7 mars 2001 , lors de la $744^{\mathrm{e}}$ réunion des délégués des ministres.

4. Adoptée le 19 juin 2003 , lors de la $844^{\mathrm{e}}$ réunion des délégués des ministres.

5. Adoptée le 25 juin 2003 ( $21^{\mathrm{e}}$ séance).

6. Adoptée le 19 mai 2004 , lors de la $884^{\mathrm{e}}$ réunion des délégués des ministres.

7. Adoptée le 23 janvier 2013 ( $6^{\mathrm{e}}$ séance).

8. Elle est entrée en vigueur le $1^{\text {er }}$ décembre 1999 et enregistre, au 29 juillet 2016,29 ratifications.

9. Elle est entrée en vigueur le $1^{\text {er }}$ février 2008 et enregistre, au 29 juillet 2016,46 ratifications.

10. Voir le $\$ 5$ de la résolution 63/14 de l'Assemblée générale de l’ONU ( Coopération entre l’Organisation des Nations unies et le Conseil de l'Europe»), adoptée le 3 novembre 2008 ( $37^{\mathrm{e}}$ séance plénière). Pour le texte de la résolution, voir Nations unies, doc. A/RES/63/14, distr. générale, 16 décembre 2008, p. 2.

11. «Résumé général de l'étude conjointe du Conseil de l'Europe et des Nations unies sur le trafic d'organes, de tissus et de cellules et la traite des êtres humains aux fins de prélèvement d'organes", $5^{\mathrm{e}}$ recommandation in fine, p. 2, en ligne: http://www.coe.int/t/dg3/healthbioethic/ Activities/05_Organ_transplantation_en/Resume_general_de_lEtude_conjointe_du_Conseil_de_l.pdf.

12. Pour le processus d'adoption de la Convention, voir Conseil de l’Europe, document CDPC (2013) 5 Rev 2, Strasbourg, 16 avril 2013 , p. 2-4, § 6-14. 
pour la bioéthique (CDBI) et le Comité européen sur la transplantation d'organes (CD-P-TO), de définir en commun les éléments essentiels susceptibles de faire partie d'une convention internationale de droit pénal contre le trafic d'organes humains, de tissus et de cellules.

Le projet de texte de la Convention du Conseil de l'Europe contre le trafic d'organes humains (ci-après «la Convention »), élaboré par le Comité d'experts sur le trafic d'organes, de tissus et de cellules humains (PC-TO), est devenu effectif avec l'approbation du CDPC intervenue en décembre 2012, et son adoption définitive par le Comité des ministres le 9 juillet 2014.

En fin de compte, la Convention a été ouverte à la signature $^{13}$ le 25 mars 2015, à Saint-Jacques-de-Compostelle (Espagne) et n'est pas encore en vigueur. Elle constitue prima facie une sorte de révolution au sein du Conseil de l'Europe en vue d'éradiquer de facto le trafic d'organes humains ${ }^{14}$, notamment en exigeant des États parties qu'ils érigent en infraction pénale le prélèvement illicite de ces organes. De surcroît, elle prévoit de manière concrète la réalisation d'un objectif de nature triple ${ }^{15}$, en l'occurrence la prévention et la lutte contre le trafic d'organes humains, la protection des droits des victimes et la facilitation de la coopération aux niveaux national et international.

L'intérêt donc d'examiner ce traité a pour finalité d'évaluer in globo les mesures et moyens envisagés pour combattre un tel trafic. En d'autres termes, il sied de savoir en quoi consiste l'originalité de cet instrument de nature obligatoire consacré exclusivement à la question du trafic d'organes humains. Pour répondre à cette problématique, nous aborderons, tout d'abord, les aspects originaux de la Convention (I), ensuite, le suivi fragile de l'application de la Convention (II).

\section{Les aspects originaux de la Convention}

La particularité de la Convention de Saint-Jacques-deCompostelle repose sur deux aspects significatifs: la pénalisation des actes illicites de trafic d'organes humains (A), ainsi que les mesures de protection et de prévention des victimes (B).

\section{A. La pénalisation des actes illicites de trafic d'organes humains}

La Convention contre le trafic d'organes humains s'applique «au trafic d'organes humains à des fins de transplantations ou à d'autres fins, et à d'autres formes de prélèvement illicite et d'implantation illicite ${ }^{16}$. De ce fait, les États parties sont tenus de sanctionner pénalement conformément à leur droit interne l'ensemble des actes de prélèvement illicite envisagés à l'article 4, alinéa 1, de la Convention. De manière concrète, ils doivent réprimer le prélèvement d'organes humains de donneurs vivants ou décédés dans trois situations ${ }^{17}$ : l'absence de consentement libre, éclairé et spécifique ou l'absence d'autorisation en vertu du droit interne; l'octroi ou l'obtention d'un profit ou d'un avantage comparable en échange du prélèvement d'organes sur un donneur vivant ou sur un donneur décédé. L'expression "un profit ou un avantage comparable» ne couvre pas, suivant l'alinéa 3 de l'article 4 , l'indemnisation du manque à gagner ou les dépenses justifiables causées par le prélèvement. L'inclusion de cette formule prévue par le Protocole additionnel à la Convention sur les droits de l'homme et la biomédecine relatif à la transplantation d'organes et de tissus d'origine humaine ${ }^{18}$ s'explique par la volonté des promoteurs de la Convention d'opérer un distinguo «entre l'indemnisation licite pouvant être accordée à des donneurs d'organes dans certains cas et la pratique interdite consistant à tirer des bénéfices financiers du corps humain et de ses différentes parties $»^{19}$.

La sanction concerne aussi le prélèvement d'organes humains de donneurs vivants ou décédés, s'il est effectué en dehors du système interne de transplantation ou en violation des préceptes internes régissant la matière (art. 4, al. 4); l'utilisation d'organes prélevés de manière illicite à des fins d'implantation ou à d'autres fins que l'implantation (art. 5) ; l'implantation d'organes hors du système interne de transplantation ou en violation des principes essentiels des lois nationales en matière de transplantation (art. 6); la sollicitation et le recrutement d'un donneur ou d'un receveur d'organes en vue d'un profit ou d'un avantage comparable pour la personne qui sollicite ou recrute ou pour une tierce personne (art. 7, al. 1). Il

13. À cette occasion, le Conseil de l'Europe a organisé en coopération avec les autorités espagnoles une conférence internationale de haut niveau contre le trafic d'organes humains. Voir le document de réflexion (http://www.coe.int/t/dghl/standardsetting/cdpc/conference/Concept\%2o Paper_Fin_FR.pdf) et les conclusions (www.coe.int/cdpc) du CDPC en date respectivement du 5 mars 2015 et du 14 avril 2015.

14. Conformément à l'article 2, alinéa 2, de la Convention, il faut comprendre par cette expression «une partie différenciée du corps humain, constituée de différents tissus, qui maintient, de façon largement autonome, sa structure, sa vascularisation et sa capacité à exercer des fonctions physiologiques; une partie d'organe est également considérée comme un organe si elle est destinée à être utilisée aux mêmes fins que l'organe entier dans le corps humain, les critères de structure et de vascularisation étant maintenus ».

15. Voir art. 1, al. 1, a, b et c, de la Convention.

16. Art. 2, al. 1, de la Convention.

17. Voir art. 4, al. 1, a, b et c, de la Convention.

18. Voir art. 21 («Interdiction du profit»). Ce protocole (STCE nº 186 ) est entré en vigueur le $1^{\text {er }}$ mai 2006 ; il énonce des principes généraux tels que la transparence dans l'attribution des organes et tissus et l'accès équitable des patients aux services de transplantation.

19. Conseil de l'Europe, «Rapport explicatif de la Convention du Conseil de l'Europe contre le trafic d'organes humains», Saint-Jacques-deCompostelle, 25 mars 2015, STCE $n^{\circ}$ 216, p. 7, \$39, en ligne: https://rm.coe.int/CoERMPublicCommonSearchServices/DisplayDCTMContent? documentId $=090000168 \mathrm{ood}_{3} 8 \mathrm{cf}$. 
s'agit essentiellement «de criminaliser les activités de personnes qui servent d'intermédiaire entre les donneurs, les receveurs et les membres du personnel médical et qui les mettent en relation $»^{20}$. L'article 7 , alinéas 2 et 3 , ajoute explicitement la corruption touchant les professionnels de la santé, les fonctionnaires ou les personnes travaillant pour une entité du secteur privé, lorsqu'une telle corruption consiste à procéder au prélèvement ou à l'implantation d'un organe humain.

La préparation, la préservation, le stockage, le transport, le transfert, la réception, l'importation et l'exportation d'organes humains prélevés de manière illicite doivent être considérés au sens de l'article 8 de la Convention comme étant une infraction pénale. La répression de cet acte comme ceux précités reste conditionnée au critère de l'«intention »; effectivement, la commission de l'ensemble de ces actes doit être causée «intentionnellement». Sur ce dernier point, le rapport explicatif du Conseil de l'Europe ayant trait à la Convention précise que l'interprétation de cette notion

[...] est laissée à l'appréciation du droit interne, mais l'exigence d'une conduite intentionnelle porte sur tous les éléments de l'infraction. Comme toujours dans les conventions pénales du Conseil de l'Europe, cela ne signifie pas que les Parties ne sont pas autorisées à aller au-delà de cette exigence minimale en criminalisant aussi des actes non intentionnels ${ }^{21}$.

Dans le même ordre d'idées, le droit pénal matériel de la Convention impose aux États parties de réprimer tout acte de complicité et toute tentative visant l'accomplissement des actes mentionnés ci-dessus (art. 9). La criminalisation de ces deux types d'actes ne sera effective que s'ils sont commis intentionnellement.

La compétence des États parties à l'égard des infractions envisagées par la Convention est régie par l'article 10. Celui-ci prévoit à son alinéa $1(\mathrm{a}, \mathrm{b}, \mathrm{c}, \mathrm{d}, \mathrm{e})$ un certain nombre de critères sur lesquels se fonde cette compétence: le territoire autorisant chaque État à sanctionner les infractions commises sur son sol; la nationalité par laquelle chaque État se déclare compétent afin de juger audelà de sa sphère interne les infractions commises par ses ressortissants à l'étranger; la résidence habituelle suivant laquelle chaque État est compétent pour connaître des infractions commises à l'étranger par les personnes ayant leur résidence habituelle sur son propre territoire. Par ailleurs, la compétence de chaque État s'étend aux infractions commises à bord de navires battant son pavillon ou d'aéronefs immatriculés conformément à sa législation.

La compétence de chaque État partie à la Convention contre le trafic d'organes humains s'établit en vertu de l'article 10, alinéa 2, à l'encontre de l'un de ses nationaux ou d'une personne ayant sa résidence habituelle sur son territoire. Elle reste absolue du fait que l'engagement des poursuites n'est pas conditionné à une plainte de la victime ou d'une dénonciation de l'État du lieu de la commission de l'infraction.

La responsabilité des personnes morales s'agissant des infractions contenues dans la Convention est réglementée par les dispositions de l'article 11. L'alinéa 1 de ce dernier énonce les conditions permettant d'engager la responsabilité d'une personne morale, à savoir la commission d'une infraction prévue par la Convention; la perpétration de cette infraction au profit d'une personne morale; l'imputation de l'infraction à une personne exerçant un pouvoir de direction; l'agissement de cette dernière au nom de la personne morale en tant que détenteur d'un pouvoir de représentation, un pouvoir de décision ou un pouvoir de contrôle. La responsabilité d'une personne morale peut revêtir trois formes: pénale, civile ou administrative. Les sanctions à l'encontre des personnes morales doivent être "effectives, proportionnées et dissuasives " ${ }^{22}$, comprenant des sanctions pécuniaires pénales ou non pénales, ainsi que d'autres mesures complémentaires telles que l'interdiction temporaire ou définitive d'exercer une activité commerciale, le placement sous surveillance judiciaire et la dissolution (art. 12, al. 2).

L'inclusion par les promoteurs de la Convention de dispositions consacrées aux personnes morales tient compte de la gravité des infractions liées au trafic d'organes humains; en effet, "le but est de faire en sorte que la responsabilité de sociétés commerciales, d'associations et de personnes morales similaires puisse être engagée en cas d'action criminelle commise pour leur compte par toute personne exerçant un pouvoir de direction en leur sein ${ }^{23}$.

Pour le reste, la Convention de Saint-Jacques-deCompostelle prévoit pareillement d'autres moyens pour la répression des infractions pénales par le biais de la saisie et la confiscation des produits de ces infractions, ou de biens d'une valeur équivalente à ceux-ci, ainsi que la fermeture temporaire ou définitive de tout établissement utilisé pour commettre de telles infractions (art. 12, al. 3). L'obligation pour tout État partie de tenir compte de certaines circonstances aggravantes dans la détermination des peines relatives aux infractions établies en vertu de la Convention est envisagée par l'article 13. Il s'agit des cas où l'infraction concernée a causé le décès de la victime ou a porté gravement atteinte à sa santé physique ou mentale, ou a été commise par des personnes abusant de la confiance que leur confère leur position, par une organisation criminelle ou à l'encontre d'un enfant ou de toute autre personne particulièrement vulnérable. De surcroît, dans l'appréciation de la peine, les condamnations

20. Conseil de l’Europe, «Rapport explicatif de la Convention...», p. 9, $\$ 52$.

21. Ibid., p. $5, \$ 28$.

22. Il en est de même pour les personnes physiques qui encourent des sanctions privatives de liberté pouvant donner lieu à l'extradition (art. 12, al. 1 in fine).

23. Conseil de l'Europe, «Rapport explicatif de la Convention...», p. 12, $₫ 76$. 
définitives prononcées dans un autre État partie sont prises en compte (art. 14).

La poursuite et l'ouverture d'une enquête à propos des infractions définies dans la Convention ne sont pas conditionnées au dépôt de plainte par la victime. Le retrait de la plainte ne met pas fin à la procédure engagée (art. 15) d'autant plus que les États parties sont tenus de garantir des enquêtes et des poursuites pénales efficaces (art. 16).

\section{B. Les mesures de protection et de prévention des victimes}

La Convention contre le trafic d'organes humains consacre en son article 3 un principe fondamental dans la jouissance des mesures concernant la protection et la prévention des victimes ${ }^{24}$, en l'occurrence le principe de non-discrimination. Cette jouissance doit être garantie

[...] sans discrimination aucune fondée notamment sur le sexe, la race, la couleur, la langue, l'âge, la religion, les opinions politiques ou toute autre opinion, l'origine nationale ou sociale, l'appartenance à une minorité nationale, la fortune, la naissance, l'orientation sexuelle, l'état de santé, le handicap ou toute autre situation. (art. 3$)^{25}$

Tenant compte des effets néfastes du trafic d'organes humains pour les victimes, les promoteurs de la Convention ont jugé utile de préconiser des dispositions afférentes à la protection des victimes. Ainsi, chaque État partie doit garantir à celles-ci l'accès aux informations pertinentes se rapportant à leur situation et qui sont nécessaires à la protection de leur santé et d'autres droits concernés, l'assistance dans leur rétablissement physique, psychologique et social, et surtout, leur indemnisation par les auteurs d'infractions pour les préjudices subis (préjudice moral et préjudice matériel) (art. 18, al. a, b et c). De plus, au cours de l'enquête et de la procédure pénale, les victimes ont le droit d'être informées de leurs droits et des services qui sont à leur disposition et, à leur demande, des suites données à leur plainte, des chefs d'accusation retenus et de l'état de la procédure pénale; d'être entendues, de présenter des éléments de preuve et de voir leur avis, leurs besoins et leurs préoccupations présentés, directement ou par le biais d'un intermédiaire, et pris en compte; d'être protégés ainsi que leur famille contre l'intimidation et les représailles (art. 19, al. 1). Cette protection s'étend également aux victimes témoins et à leurs proches (art. 20).
Dans une optique similaire, le statut des victimes dans les procédures pénales se voit conforté à travers un certain nombre de mesures devant être prises par les États parties: l'accès aux informations sur les procédures judiciaires et administratives pertinentes; l'accès à une assistance judiciaire gratuite; la possibilité de porter plainte auprès des autorités compétentes de leur État de résidence; la possibilité pour des groupes, fondations, associations ou organisations gouvernementales ou non gouvernementales d'assister et / ou d'aider les victimes (art. 19, al. 2, 3, 4 et 5).

Pour ce qui est des mesures de prévention, la Convention contre le trafic d'organes humains comprend deux volets. Le premier volet porte sur les mesures de prévention au plan national: les États parties doivent assurer l'existence d'un système interne transparent pour la transplantation d'organes humains ${ }^{26}$, garantir aux patients un accès équitable aux services de transplantation ${ }^{27}$ et assurer la collecte, l'analyse et l'échange d'informations sur les infractions ayant trait au trafic d'organes humains (art. 21, al. 1). Pour rendre ces mesures efficaces, chaque État partie est appelé à conforter la formation des professionnels de santé et des agents concernés par les informations sur la prévention du trafic d'organes humains; à organiser des campagnes de sensibilisation du public à l'illégalité et aux dangers du trafic d'organes humains; à interdire la publicité sur le besoin d'organes humains, ou sur leur disponibilité, en vue d'offrir ou de rechercher un profit ou un avantage comparable (art. 21, al. 2 et 3 ).

Quant au second volet, il concerne les moyens de prévention au niveau international relevant entièrement de la coopération des États parties. Ceux-ci doivent informer par le truchement d'un rapport le Comité des parties du nombre de cas de trafic d'organes humains sur leur territoire respectif et désigner un point de contact national responsable de l'échange d'informations entre eux.

L'originalité de la Convention sur le plan substantiel ne doit pas pour autant masquer la fragilité du système de contrôle préconisé.

\section{Le suivi fragile de l'application de la Convention}

La Convention contre le trafic d'organes humains préconise un système de contrôle fragile pour assurer l'application de ses dispositions. Cette fragilité résulte de la pluralité des mécanismes de suivi (A), ainsi que de certaines déficiences relatives au système de suivi (B).

24. La notion de victime n'est pas définie par la Convention. Elle relève de l'appréciation des États parties.

25. Voir l'article 14 de la Convention de sauvegarde des droits de l'homme et des libertés fondamentales (Convention EDH). En pratique, la Cour européenne des droits de l'homme a précisé, au sujet de la portée générale de l'article 14, qu'une distinction est discriminatoire si elle manque de justification «objective et raisonnable» (affaires Abdulaziz, Cabales et Balkandali c. Royaume-Uni, nº 9214/80 9473/81 9474/81 du 28 mai 1985 et Karlheinz Schmidt c. Allemagne, no $13580 / 88$ du 18 juillet 1994).

26. Cette transparence contribue à réduire «le risque que des organes prélevés de manière illicite soient introduits dans le système légal de transplantation» («Rapport explicatif de la Convention...», p. 20, §126).

27. Cet accès «signifie que les Parties doivent traiter tous les patients en attente d'un organe sur un pied d'égalité lors de l'affectation des organes» («Rapport explicatif de la Convention...», p. 20, §126). 


\section{A. La pluralité des mécanismes de suivi}

La mise en œuvre de la Convention contre le trafic d'organes humains incombe essentiellement au Comité des parties, organe composé de représentants des États parties à cet instrument (art. 23, al. 1). Au secrétaire général du Conseil de l'Europe revient le droit de convoquer la première réunion du Comité des parties, dans un délai d'un an à compter de l'entrée en vigueur de la Convention pour le dixième signataire l'ayant ratifiée ${ }^{28}$. Par la suite, il se réunira à la demande d'au moins un tiers des États parties ou de l'organe administratif de l'institution strasbourgeoise (art. 23, al. 2).

Dans l'exercice de sa mission de contrôle ${ }^{29}$, le Comité des parties fixe à travers son règlement intérieur la procédure d'évaluation de la Convention en favorisant une approche plurisectorielle et pluridisciplinaire. De ce fait, il assure "la collecte, l'analyse et l'échange d'informations, d'expériences et de bonnes pratiques entre les États afin de renforcer leur capacité à prévenir et à lutter contre le trafic d'organes humains» (art. 25, al. 2). Par ailleurs, le Comité des parties assume, conformément aux dispositions de l'article 25, alinéa 3 ( $a, b, c)$ de la Convention, trois principales fonctions consistant à :

- formuler des propositions concrètes garantissant l'application effective de la Convention, y compris en identifiant les effets des déclarations ou réserves faites au titre de l'instrument en question;

- donner un avis consultatif sur toute question ayant trait à l'application de la Convention, en y incluant l'échange d'informations entre les États parties s'agissant des aspects juridiques, politiques ou techniques liés à la Convention;

- adresser des recommandations aux États parties au sujet de l'application de la Convention.

À côté du Comité des parties - principal organe de contrôle -, la Convention contre le trafic d'organes humains préconise d'autres mécanismes de suivi (art. 24), en vue de concrétiser l'approche plurisectorielle et pluridisciplinaire tant voulue par les promoteurs de l'instrument européen. Le Comité européen pour les problèmes criminels $(\mathrm{CDPC})^{30}$ occupe une place assez spéciale parmi ces mécanismes. Effectivement, il revient à lui seul, conformément à l'article 25, alinéa 4, d'être informé du fonctionnement global du Comité des parties et de sa fonction de contrôle. De surcroît, il est étroitement associé au règlement amiable de tout différend pouvant naître de la mise en œuvre des dispositions de la Convention (art. 31).

L'architecture du système de contrôle de la Convention implique aussi la participation de l'Assemblée parlementaire du Conseil de l'Europe (APCE) ${ }^{31}$ dont le rôle consiste, entre autres, à formuler des avis sur les instruments adoptés sous l'égide de l'institution strasbourgeoise et à mener des enquêtes sur les violations des droits de l'homme; du Comité de bioéthique (DH-BIO) chargé de la Convention pour la protection des droits de l'homme et de la dignité de l'être humain à l'égard des applications de la biologie et de la médecine ${ }^{32}$, ainsi que du Comité européen sur la transplantation d'organes (CD-P-TO) ${ }^{33}$ en charge des questions se rapportant à la transplantation d'organes au sein de la Direction européenne de la qualité du médicament (EDQM).

En pratique, l'efficacité du mécanisme de suivi de la Convention se voit confortée à travers la participation ${ }^{34}$ aux travaux du Comité des parties, à titre d'observateurs, des représentants d'organes internationaux pertinents, ceux d'organes officiels pertinents des parties et des organisations non gouvernementales (art. 24, al. 3, 4 et 5). Dans un registre semblable, les États parties peuvent contribuer à l'application des dispositions de la Convention par le truchement d'accords bilatéraux ou multilatéraux conclus entre eux (art. 26, al. 2).

\section{B. Les déficiences afférentes au système de suivi}

La fébrilité du système de contrôle préconisé par la Convention contre le trafic d'organes humains résulte également d'un certain nombre d'aspects liés au fonctionnement du mécanisme dans sa globalité. En réalité, le mécanisme a été grippé dès le départ; en effet, le contrôle des obligations juridiques des États parties contractées au titre de la Convention incombe à un organe de nature politique, le Comité des parties. De ce fait, les États peuvent de mauvaise foi ne pas vouloir contribuer in concreto à l'application des dispositions de la Convention. Il aurait été souhaitable d'envisager un comité ou un groupe d'experts

28. Il faut remarquer que «les négociateurs ont essayé de faire en sorte que la Convention entre en vigueur rapidement, tout en différant l'introduction du mécanisme de suivi jusqu'à ce que la Convention soit ratifiée par un nombre suffisant d'États pour pouvoir fonctionner dans de bonnes conditions, avec un nombre suffisant de Parties représentées pour garantir sa crédibilité» («Rapport explicatif de la Convention... », p. 22, \$134).

29. En vertu de l'article 23, alinéa 4, de la Convention, le Comité des parties sera assisté par le secrétariat du Conseil de l’Europe.

30. Institué en 1958 par le Comité des ministres conformément à l'article 17 du Statut du Conseil de l'Europe. Il comprend des représentants des États membres du rang le plus élevé et est responsable du contrôle et de la coordination des activités du Conseil de l'Europe en matière de prévention et de contrôle du crime. Voir, pour son mandat, le lien suivant: http://www.coe.int/t/dghl/standardsetting/cdpc/CDPC\%20documents/Mandat\%20 CDPC\%202016-2017.pdf.

31. C'est la conscience démocratique de l'Europe. Elle comprend 318 parlementaires des 47 États membres du Conseil de l'Europe.

32. Adoptée par le Comité des ministres, le 19 novembre 1996. Elle a été complétée par quatre protocoles additionnels.

33. Créé en 1987, à l'occasion de la $3^{\text {e }}$ conférence des ministres européens de la santé relative aux aspects éthiques, organisationnels et juridiques de la transplantation d'organes.

34. Cette participation «assure une représentation équilibrée des différents secteurs et disciplines impliqués (autorités d'application de la loi, système judiciaire, autorités en matière de santé et groupes d'intérêts de la société civile)» («Rapport explicatif de la Convention...», p. 23, §141) 
indépendants chargé de la mise en œuvre des dispositions de la Convention à l'instar du système onusien de protection des droits de l'homme. À l'extrême, on aurait même dû suivre l'exemple de la Convention du Conseil de l'Europe sur la lutte contre la traite des êtres humains ${ }^{35}$ prévoyant, conformément à son article 36, un Groupe d'experts sur la lutte contre la traite des êtres humains (GRETA) ${ }^{36}$.

Un autre aspect susceptible d'affecter sérieusement le système de suivi préconisé tient à la clause de réserve contenue dans de nombreuses dispositions de la Convention permettant aux États parties de se soustraire à leurs obligations juridiques. C'est le cas, par exemple, des articles 4, alinéa 2 (prélèvement illicite d'organes humains), 9, alinéa 3 (complicité et tentative), 10, alinéas 3 et 5 (compétence), 30, alinéa 2 (réserves) conférant à tout État ou à l'Union européenne un pouvoir d'appréciation large quant à leur mise en œuvre par la formulation d'une déclaration adressée à cette fin au secrétaire général du Conseil de l'Europe. Ces nombreuses clauses échappatoires concédées aux États parties ne font que limiter la portée réelle de l'instrument européen et partant le vider de sa teneur. C'est pourquoi il eut été de bon sens d'exclure la formulation de réserves, surtout que la Convention porte sur un phénomène universel, le trafic d'organes humains. À cet égard, la Convention du Conseil de l'Europe sur la lutte contre la traite des êtres humains exclut toute réserve aux dispositions de la Convention, hormis celle prévue à l'article 3 , alinéa 2 (compétence).

Par ailleurs, il sied d'ajouter un autre facteur illustrant à souhait cette fragilité, en l'occurrence la liberté dont disposent les États parties pour ériger en infractions pénales ${ }^{37}$ les actes visés dans le chapitre II de la Convention relatif au droit pénal matériel; en effet, ils ne peuvent le faire que si lesdits actes sont commis de manière «intentionnelle» - celle-ci relève plus du droit interne des États parties. D'autant plus que les articles ${ }^{38}$ concernés par le chapitre en question utilisent maladroitement l'expression «mesures législatives et autres nécessaires». Dans un contexte similaire, l'article 20, alinéa 1, de la Convention (protection des témoins) permet aux États parties de mettre en avant l'argument «selon les moyens à sa disposition et conformément aux conditions définies par son droit interne», pour ne pas assurer une protection efficace des témoins contre tous actes de représailles ou d'intimidation.

L'ensemble de ces échappatoires ne fait qu'adoucir la responsabilité des États parties eu égard à leurs obligations juridiques et fragilise davantage le système de contrôle.

L'adoption par le Conseil de l'Europe de la Convention contre le trafic d'organes humains tombe à point nommé pour lutter efficacement contre ce fléau planétaire. Les efforts déployés par l'institution strasbourgeoise ont finalement abouti à un instrument audacieux et révolutionnaire de nature juridique obligatoire pour la prévention et la répression du commerce illicite de la personne humaine. L'originalité de l'instrument européen repose entièrement sur deux éléments intrinsèques: la pénalisation des actes illicites de trafic d'organes humains, ainsi que la prévention et la protection des personnes victimes de ces actes.

Nonobstant cette originalité, la Convention contre le trafic d'organes humains - texte de compromis - suscite tout de même un certain nombre d'interrogations; en effet, n'est-il pas quelque part contradictoire de mettre l'accent sur le droit pénal matériel, alors que simultanément les États parties bénéficient d'une grande latitude quant à la mise en œuvre de ses dispositions? Dans le même ordre d'idées, n'est-il pas étonnant de prévoir uniquement cinq instruments de ratification (art. 28) pour assurer de facto son entrée en vigueur ${ }^{39}$, alors que la Convention tend à l'universalité en prévoyant une large adhésion (États membres du Conseil de l'Europe et ceux non membres ayant le statut d'observateur auprès de celui-ci et de l'Union européenne)?

En vérité, la clause relative aux réserves contenue dans certaines dispositions de la Convention et les clauses échappatoires concédées aux États membres risquent sérieusement d'affaiblir et de limiter en pratique la portée dudit traité. Il en est de même pour la fragilité du mécanisme de suivi de la Convention. Au demeurant, la mise en œuvre de la Convention contre le trafic d'organes humains dépend largement de la coopération des États et des moyens financiers mis à disposition de l'organe de contrôle.

35. L'article 37 de cette Convention prévoit aussi un Comité des Parties (organe politique).

36. Il se compose de 10 membres au minimum et de 15 membres au maximum (art. 36, al. 2, de la Convention du Conseil de l'Europe sur la lutte contre la traite des êtres humains)

37. Voir, par exemple, art. 4, 7, 8 et 9 de la Convention.

38. Ibid.

39. Au 29 juillet 2016, la Convention enregistre 1 ratification et 15 signatures. 